\title{
Trabajo colaborativo entre docentes e investigadores en Didáctica de la Matemática: de la reflexión sobre las prácticas a la elaboración de ejes de análisis para la enseñanza ${ }^{1}$
}

Collaborative work between teachers and researchers in Mathematics Education: from reflection on practices to the elaboration of analysis axes for teaching

\author{
Patricia Sadovsky ${ }^{2}$ \\ Horacio Itzcovich ${ }^{3}$ \\ María Mónica Becerril ${ }^{4}$ \\ María Emilia Quaranta ${ }^{5}$ \\ Patricia García ${ }^{6}$
}

Resumen: En este artículo se comparten resultados de una investigación que se propone estudiar las elaboraciones que tienen lugar cuando docentes e investigadores en didáctica de la matemática colaboran para analizar problemas de enseñanza. Las discusiones sostenidas con un grupo de maestras

Fecha de recepción: 29 de agosto de 2018. Fecha de aceptación: 4 de marzo de 2019.

1 Los resultados de este trabajo surgen de una investigación desarrollada por los autores en el marco de la programación 2016-2017 de la Universidad Pedagógica Nacional UNIPE.

2 Departamento de Ciencias y Tecnología, Universidad Pedagógica Nacional (Unipe), Buenos Aires, Argentina, patsadov@gmail.com, orcid.org/0000-0001-6703-3315

${ }^{3}$ Departamento de Ciencias y Tecnología, Universidad Pedagógica Nacional (Unipe), Buenos Aires, Argentina, yayohiz@gmail.com, orcid.org/0000-0003-4189-8593

${ }^{4}$ Departamento de Ciencias y Tecnología, Universidad Pedagógica Nacional (Unipe), Buenos Aires, Argentina, monicabece@gmail.com, orcid.org/0000-0002-3922-447X

${ }^{5}$ Departamento de Ciencias y Tecnología, Universidad Pedagógica Nacional (Unipe), Buenos Aires, Argentina, memiliaquaranta@gmail.com, orcid.org/0000-0002-3760-2369

${ }^{6}$ Departamento de Ciencias y Tecnología, Universidad Pedagógica Nacional (Unipe), Buenos Aires, Argentina, patgarcia41@speedy.com.ar, orcid.org/0000-0002-6247-0310 
y la directora de una escuela pública del conurbano bonaerense posibilitaron la producción de un conjunto de ideas que organizamos alrededor de tres dimensiones de análisis: las relaciones entre las interacciones personales y grupales del docente con los alumnos, los distintos planos en los que intervienen las ayudas que las maestras pueden brindar a sus alumnos y las mediaciones necesarias para favorecer la disponibilidad de recursos y/o conocimientos. En el marco de las discusiones se hace visible el par decisión de enseñanzarazones que la sostienen como base de la construcción de criterios que orientan la acción docente. Poco a poco un estado inicial de incertidumbre en el que se piensan las maestras va dando lugar a un reconocimiento de la complejidad de la enseñanza, cuando se opta por incorporar los aportes de los alumnos al tratamiento de los conceptos matemáticos en sala de clase.

Palabras claves: Trabajo colaborativo entre investigadores y docentes - Dimensiones de análisis de la enseñanza - Complejidad de la acción didáctica

\begin{abstract}
Results from a research, which studies elaborations that take place when teachers and researchers in mathematics education collaborate to analyse teaching problems, are shared in this paper. Discussions held with a group of teachers and the head of a primary school in Buenos Aires suburbs gave rise to some elaborations. Three dimensions of analysis support them: relationships between personal and group interactions teachers and students hold, different levels in which teachers can help their students to contribute to learning progress and mediations to make certain knowledge available as a resource. Within the framework of these discussions, it becomes visible the pair decision to teach - reasons that support it as a basis for the construction of some criteria to guide teaching action. Little by little, an initial state of uncertainty in which teachers think about themselves is leading to an acknowledgment of the complexity of teaching when they decide to incorporate students' contributions into the treatment of mathematical concepts in the classroom.
\end{abstract}

Keywords: Collaborative work between researchers and teachers - Dimensions for teaching analysis - Complexity of the didactic action 


\section{INTRODUCCIÓN}

Los resultados que compartimos en este artículo fueron elaborados en el marco de un proyecto de investigación que se viene desarrollando desde el año 2012 bajo el encuadre de trabajo colaborativo entre investigadores y docentes (Bednarz, 2013; Proulx, 2013; Sensevy, 2011). Como lo hemos comunicado en trabajos previos (Sadovsky, P., Quaranta, M.E.; Itzcovich, H.; Becerril, M.M.; García, P., 2015; Sadovsky, P.; Itzcovich, H.; Quaranta, M., Becerril, M.; García, P., 2016), nuestro grupo toma como objeto de estudio las ideas matemático-didácticas que se van elaborando en los intercambios que tienen lugar en espacios de discusión en los que integrantes de nuestro equipo se reúnen periódicamente con grupos de maestros de escuela primaria en sus lugares de trabajo, para analizar de manera conjunta cuestiones vinculadas a la enseñanza de la matemática. Los asuntos que van estructurando las conversaciones surgen de preocupaciones que atañen a la tarea de enseñanza que enfrentan los maestros y también, a medida que el grupo colaborativo se va enriqueciendo, de las propias elaboraciones que se van haciendo en su seno. En ambos planos los investigadores que coordinan los grupos se proponen construir y sostener posiciones simétricas entre los integrantes (Sadovsky, Quaranta, Itzcovich, Becerril, García, 2015; Sensevy, 2011). Nuestra investigación tiene dos grandes focos: los desarrollos del grupo que comparte con los maestros y el proceso de construcción de la colaboración en tanto el mismo supone una problematicidad ligada a históricas divisiones sociales del trabajo entre el mundo académico y el mundo de las prácticas de enseñanza que se refleja, entre otros aspectos, en los modos de enfocar los problemas, en los puntos de apoyo para las conclusiones a las que se arriba, en los tiempos disponibles para los análisis, en las responsabilidades que se asumen, en las referencias a los grupos profesionales de pertenencia y a los grupos sociales que ejercen control sobre el trabajo (Robert, 2003; Sadovsky, P.; Itzcovich, H.; Quaranta, M., Becerril, M.; García, P., 2016).

El grupo de colaboración se reúne periódicamente en la escuela. Allí se van abordando cuestiones de enseñanza de la matemática, en general propuestas por los maestros porque les resultan problemáticas y para las cuales se suelen delinear de manera conjunta modos de recoger material en las aulas (producciones de alumnos, registros de episodios de clases), que alimente la discusión. La decisión de sostener los intercambios sobre la base de esta documentación obedece a la intención de transformar aquello que se produce en las aulas en 
objeto de análisis y tener así una base empírica para reconstituir la matemática que allí se enseña.

Cada reunión es registrada en audio y, a partir de la desgrabación, el equipo de investigadores que asiste a la escuela realiza una síntesis que se lee al comienzo de la reunión siguiente. Esto permite retomar los temas, revisar las ideas tratadas e ir objetivando la discusión. Una condición metodológica fundamental es que tanto docentes como investigadores conciban el espacio de trabajo compartido como un ámbito en el que se construirán nuevas respuestas para las preguntas que se abordan. Esta condición no se realiza simplemente con la voluntad declarada de sostenerla sino que es producto de una construcción en el tiempo, en la que también intervienen la confianza que se va construyendo así como la reflexión sostenida en el grupo de investigadores sobre la marcha de la colaboración. Obviamente no se trata de respuestas completamente nuevas ya que provienen del bagaje conceptual de cada uno de los integrantes del espacio. Más bien, lo que queremos enfatizar es que ponemos cuidado en no proponer, sobre todo los investigadores, un repertorio de recursos ya acuñados que muchas veces no toman en cuenta el contexto particular que se analiza.

En este artículo damos cuenta de los procesos desarrollados en un caso, ${ }^{7}$ el grupo de la Escuela 30 de Longchamps ${ }^{8}$ en el que participaron las maestras de primero a cuarto grado, la directora y dos integrantes de nuestro equipo, que se reunieron periódicamente durante los años 2016, 2017 y 2018. Nos proponemos trabajos de largo plazo atendiendo a dos razones básicas: porque pensamos que es un modo de construir una historia de las ideas que se van produciendo, de retomarlas, revisarlas e ir resignificándolas y porque la constitución de la colaboración, como se ha enunciado, es subsidiaria de tiempos prolongados.

7 La investigación asume un abordaje cualitativo e interpretativo y toma elementos de la metodología de estudios de casos. El caso se concibe como el conjunto de interacciones que se producen entre investigadores de nuestro equipo, docentes y directivos de una escuela.

8 Se trata de una institución que en el año 2014 se transformó en escuela de jornada completa. La directora tiene una decisiva orientación hacia el trabajo pedagógico y es consultada por las maestras para quienes es referente en cuestiones didácticas. Reconocemos y agradecemos muy especialmente al equipo docente de la Escuela 30 de Longchamps, Almirante Brown, Provincia de Buenos Aires, su apertura al trabajo colaborativo, su compromiso y participación: Gisela Arce, Viviana Brancatti, Adriana De Lera, Lorena Navarrete, Flavia Romani, Liliana Torres, Alejandra Quercia, Paula Pérez. 
De manera global, señalamos como resultado de esta investigación que los análisis Ilevados a cabo en el espacio de trabajo colaborativo fueron haciendo visible para todos los integrantes del grupo aspectos de la enseñanza -entramados entre sí- que se vuelven problemáticos cuando se acepta que la discusión sobre el conocimiento en el aula está afectada por las intervenciones de los alumnos. De esos aspectos damos cuenta en este artículo.

Una vez puesto a funcionar el grupo, las maestras declaran encontrarse en transición. Sus primeras intervenciones dan la impresión de que piensan que hay un antes, que ha configurado sus prácticas ligadas a la enseñanza de procedimientos convencionales de cálculo -y a las que los alumnos se han adaptado- y un ahora que no se termina de atrapar y que las envuelve en una situación de incertidumbre con relación a las decisiones que toman en las aulas.

En este primer momento la transición se describe enfatizando dos polos: un sistema de enseñanza, que sería el históricamente instalado y que las ha estructurado, y lo que ahora se trata de pensar, caracterizado por tres elementos básicos: dar lugar a diferentes procedimientos, ponerlos en común y aceptar los errores. A lo largo de las discusiones, esta mirada inicial se va abriendo -y enriqueciendo- para dar lugar a más elementos que suman complejidad a los procesos de enseñanza que se ven llamadas a sostener. Si bien este estado de transición les genera incertidumbre, sus argumentaciones reafirman su convicción de profundizar los cambios que van realizando.

Aunque parece prevalecer una idea de ruptura con las tradiciones de enseñanza, las diferentes dimensiones de análisis que van emergiendo en las discusiones a lo largo del desarrollo del espacio colaborativo dan cuenta de que sus propias historias de trabajo docente constituyen una referencia importante a partir de la cual van elaborando transformaciones en una perspectiva mucho más dialéctica entre pasado y presente que lo que explícitamente reconocen (Del Rey, 2012; Fisher, 2016). En otros términos, lejos de una visión según la cual la innovación tiene un valor por el solo hecho de ser tal, las maestras de este grupo toman en consideración la propia experiencia desarrollada en su trabajo de enseñanza y la van haciendo funcionar como un marco que les permite repensar muchas de las intervenciones que van realizando.

Desde un inicio de los intercambios, las docentes aportaron episodios de sus clases que proponían para el análisis conjunto. En general, se trataba de situaciones en las que interactuaban con un alumno -o con varios- a raíz de las decisiones que los niños tomaban al encarar la tarea que se les había propuesto. Podríamos decir que esos intercambios estaban signados por cierto desconcierto 
de las maestras frente a producciones de niños cuyo alcance, por diferentes razones, no terminaban de entender. Subrayamos el recorte que hacen las maestras en términos de su interacción con el accionar de los niños ya que entendemos que "habla" de un esquema de acción didáctico (Pastré, Mayen, \& Vergnaud, 2006) en el que la intervención docente se estructura en función de las decisiones de los alumnos en la tarea y de la intención del docente al proponerla (Sensevy, 2011; Lave, 2001).

Si bien no estamos en condiciones de atribuirles a las maestras un uso consciente de este esquema de acción, el recorte sistemático que hacen para el análisis en las reuniones nos lleva a pensar que se trata de un grupo de docentes que se ve exigido de producir in situ intervenciones no previstas, reguladas por el accionar de los alumnos. Establecemos lazos entre la incertidumbre que estas respuestas generan y el hecho de recortarlas como asuntos que "merecen" una reflexión en el grupo. En este contexto interpretamos que la necesidad de las docentes de entender las decisiones de los niños conlleva -en principio- dos aspectos: las relaciones matemáticas implicadas en lo que el niño hace y el modo en que las incorporarán a su enseñanza.

Varias son las razones por las que un episodio llega a la mesa de trabajo por decisión de alguna maestra: una respuesta errónea, un procedimiento muy básico o anti económico, un bloqueo que pone de manifiesto que los niños no cuentan con un conocimiento que se concebía disponible, una demanda cuyas razones no terminan de entender... a medida que se analizan estas intervenciones, comienza a hacerse visible la complejidad del accionar docente cuando es un propósito explícito incorporar -integrar, transformar, extender, restringir- las producciones de los alumnos -sus ideas, sus representaciones, sus procedimientos, sus argumentos, sus creencias- a las conceptualizaciones que se van haciendo en el aula. Las divergencias, énfasis o matices que surgen en las discusiones del grupo colaborativo en lo relativo a los tipos de intervención docente -su función, su oportunidad, sus requerimientos, sus condicionamientos- y -de manera solidaria- en lo concerniente a los soportes que necesitan los niños para sostenerse y progresar en los aprendizajes, van dando lugar a una configuración de la enseñanza que nosotros organizamos en ejes de análisis, bajo el propósito de ir haciendo explícito el marco compartido que va surgiendo como resultado del trabajo colaborativo.

En los primeros intercambios, las diferencias en los puntos de vista sobre alguna cuestión eran tratadas por las maestras como "intercambios de opiniones" considerados por ellas todos complementarios y válidos. En ese momento, no se 
habían construido aún en el espacio colaborativo herramientas conceptuales que permitieran tratar dichas diferencias con base en argumentos. Al profundizar el análisis a lo largo de las reuniones con los aportes de unos y otros y, apoyados en el análisis de los materiales de las aulas, estas divergencias fueron derivando en la producción de razones que contribuían a sostener las diferentes posiciones. Tal el caso de lo ocurrido respecto de la pertinencia de hacer o no públicas para toda la clase las dificultades o errores de algún alumno o grupo de alumnos, que fue evolucionando hasta hacer posible una interpretación más conceptual de las posiciones y considerar criterios que respalden las decisiones que se toman al respecto. En esas condiciones entendemos que se pudo constituir como eje el análisis de las relaciones entre las interacciones personales y las grupales en sala de clase. Desarrollamos esta cuestión en el punto 2.

¿Qué significa ayudar a un alumno?; ¿cómo reconocer sus progresos?; ¿por qué a veces la ayuda "funciona" y otras no?; ¿por qué no siempre la apelación a algún material concreto es soporte para la resolución de un alumno que parece bloqueado?; ¿ayudar es guiarlo para que resuelva o es orientarlo para que desarrolle la idea que está poniendo en juego aunque no sea válida?; ¿es posible para el docente reconocer el progreso de sus alumnos en el marco de las ayudas que brinda?; una estrategia que se planifica para enfrentar un problema reconocido de enseñanza, ¿puede pensarse a la vez como ayuda?; ¿cómo se ayuda a los niños que parecen distantes de una intención de aprender? Estas preguntas dan cuenta de una problematización progresiva en la que se van enriqueciendo los contenidos, las estrategias y los sentidos de las ayudas a los niños. Analizamos este proceso en el punto 3.

Alentar a que los alumnos desplieguen sus propias estrategias frente a la resolución de problemas es una práctica instalada en el equipo de maestras, anterior a la constitución del grupo colaborativo. A la vez se preguntan ¿cómo impulsar su progreso?. En particular se analiza la relación entre la disposición de ciertos recursos de cálculo que podrían funcionar como mediadores y su uso específico para determinados fines. Desarrollamos las ideas vinculadas a esta cuestión en el punto 4.

Nuestras conclusiones retoman globalmente los análisis realizados bajo algunas preguntas: ¿cuáles son las grandes trazas de la producción del grupo?, ¿cómo se ha modificado el estado inicial de transición planteado por las maestras?, ¿y la incertidumbre asociada?, ¿en qué sentidos el trabajo compartido interpela las posiciones históricamente cimentadas de maestros e investigadores?, ¿qué relaciones establecemos entre la situación colaborativa y las 
transformaciones de los conocimientos de los integrantes del grupo?, ¿qué modificaciones se registran en el proceso investigativo? Se esbozan respuestas para estas cuestiones en el punto 5.

\section{2. ¿INTERVENCIONES PERSONALES O GRUPALES? DEL INTERCAMBIO DE PUNTOS DE VISTA A LA CONSTRUCCIÓN DE UN EJE DE ANÁLISIS}

Las maestras plantean inicialmente incertidumbres vinculadas al alcance de las producciones de los alumnos, sobre todo relativas a los problemas de resta: su nivel de elaboración, las ideas implicadas, la pertinencia de aceptarlas sin más o, por el contrario, de presionar para que avancen a otras de mayor complejidad. Esto deriva en el compromiso de realizar alguna actividad en las aulas y recoger trabajos de los niños, para analizar en el espacio las estrategias puestas en juego. Fue la primera tarea compartida en el grupo a la que las maestras aportaron gran cantidad de material. Surge en la discusión la siguiente cuestión: frente a las dificultades o errores de los alumnos, ¿es necesario interactuar con cada uno de ellos o podrían tratarse a través de una discusión grupal? Para algunos maestros parecían ser opciones contrapuestas. Sin embargo, no era éste nuestro punto de vista ya que, desde el enfoque didáctico que sostenemos, una decisión requiere para su análisis la explicitación de su intencionalidad y su relación con el contexto en el que se desarrolla. Reparamos así en la necesidad de ir proponiendo como proyecto para el espacio colaborativo la búsqueda de fundamentos para las decisiones que se toman. Se desarrolla a partir de este hecho una discusión que se va profundizando a lo largo del tiempo y de la que queremos dar cuenta en este apartado. Veamos.

Como mencionamos, la base de los análisis fue el material recogido en las aulas. Una de las maestras había planteado a sus alumnos el siguiente problema: Ana está juntando plata para comprarse una patineta que cuesta \$2345; ya tiene en su alcancía \$1600, ¿cuánto dinero le falta? Se reconoce la diversidad de estrategias posibles y, en particular, se valora positivamente la de sumar a 1600 su complemento respecto de 2345 que unos cuantos niños implementan. La preocupación de la maestra se centra en los chicos que suman las dos cantidades dadas y hace foco en el hecho de que los niños no controlan que obtienen un resultado inconsistente: 
M1: Claro, no está esto de analizar si es posible este resultado, más allá de que sepan o no cómo hacer, ver si es posible [el resultado].

I: Bien, ¿cómo podríamos hacer ese análisis con ellos?

M1: Para mi gusto es un trabajo individual, porque lo que piensa esta nena, lo piensa esta nena, no lo piensa un grupo.

[...]

M2: Una puesta en común sería bueno.

[...]

M1: Es esa nena, quiero saber qué piensa, dónde está. (...) A mí me parece que al grupo lo forma lo individual de cada uno, por eso apunto a eso individual, pero por conocerlo, y en base a eso, sí, la dinámica del trabajo en matemática no es lo individual, pero para este trabajo es lo individual. (...) Yo estoy de acuerdo que la puesta en común enriquece.

[...]

I: Por ahí vos planteás el procedimiento para todos y preguntás "a ver, miremos entre todos esto, ¿cómo lo habrán pensado?"

M1: Y eso, digo yo, ¿me va a dar la respuesta de dónde está el obstáculo de esta nena puntualmente? Eso es lo que me pregunto.

[...].

M2: Porque también en la soledad del problema, quizás queda ahí, pero si ella lo puede confrontar con el resto...

La maestra 2 no termina de fundamentar su propuesta de puesta en común y, la maestra 1, piensa que un trabajo en el que se discuta con todo el grupo clase las ideas que subyacen a las estrategias erróneas, no le va a permitir conocer el modo de pensar de los niños que hicieron procedimientos desajustados aunque, en términos generales, sí valora las situaciones colectivas de trabajo. Interpretamos que las diferencias radican en este momento en los focos que hacen estas maestras: en el niño, en sus dificultades, para entender por qué hace lo que hace o en el grupo clase bajo el supuesto -no necesariamente compartido por todas las integrantes del grupo- de que la discusión grupal de una idea (correcta o errónea) resulta beneficiosa para todos. Aunque esto no se llega a desarrollar en ese momento, entendemos que ya se perfilaban dos modos de ver -relevantes para nosotros desde el punto de vista didáctico- que irían cobrando mayor nitidez en el tiempo: ¿dónde está el asunto?, ¿en las dificultades del niño, en la complejidad del objeto, en una interacción entre ambas? 
Con el correr de los encuentros, estas discusiones reaparecen y se nutren con nuevos matices a través de dos grandes vías: 1) se siguen analizando las relaciones matemáticas que subyacen a diferentes estrategias de los niños y, 2) se discute acerca de su posible gestión en la clase.

Con relación a la primera de estas vías, el análisis de las estrategias en términos de conocimientos matemáticos en juego -esto es coincidente con resultados obtenidos en otros grupos con los que hemos trabajado-, contribuye a que se hagan visibles no sólo las ideas que los alumnos han utilizado sino las que deben aprender, contribuyendo a enriquecer un esquema de acción didáctica -ya señalado arriba- que coordina las ideas matemáticas de los alumnos con la intención de enseñanza. Se trata de momentos muy ricos, en los que, alrededor de una mesa, entre todas se producen verdaderas reconstrucciones acerca de cómo pudieron haber pensado los niños, se analizan las formas de representación y los recursos a los que apelaron, se comenta qué intervención tuvieron las maestras mientras los niños trabajaban, se comparan estrategias, se realizan consideraciones sobre las trayectorias de los niños y se las relaciona con sus producciones en cierto momento. Es decir, se amplía la mirada simultáneamente sobre la complejidad del objeto de enseñanza y sobre los saberes de los alumnos. La experiencia analítica de los investigadores tensa el análisis y hace visibles para las maestras sus propias posibilidades de interpretación de las acciones de los alumnos en términos de conocimiento matemático. Podemos dar cuenta entonces, de un nuevo caso que muestra que la práctica de análisis de las clases se adquiere participando con otros de la misma.

Con relación a la segunda vía de discusión que reconocemos, es decir al modo de gestionar en clase esta diversidad de estrategias, surgen un sin número de interrogantes: ¿cómo hacer para que se difundan los procedimientos "buenos"?; si un alumno pasa a narrar una estrategia, ¿es productivo que otros niños la tomen y la repliquen?; ¿se estarían "copiando"?; ¿no se inhibe de este modo que todos tengan una producción propia?; o, peor aun, ¿no se corre el riesgo de que mecanice lo que otros compañeros han pensado?; cuando surgen varias estrategias, ¿cómo seleccionamos cuáles trabajamos entre todos y cuáles sólo con los que las produjeron?, ¿̇la explicación o comprensión de los procedimientos utilizados por aquellos alumnos que se equivocaron o presentan procedimientos más básicos aportaría algo a aquellos que no cometieron esos errores o utilizan procedimientos más económicos? Estas preguntas, surgidas básicamente de las maestras abren a los ojos de nuestro equipo de investigación nuevos matices relativos a la complejidad que tiene para los maestros la gestión de las "puestas 
en común", a la que difícilmente se accedería sin este intercambio. Uno de los aspectos más ricos con relación a estas preguntas fue, desde nuestro punto de vista, arribar a la conclusión de que, más que admitir respuestas únicas o categóricas, abrir un camino de exploración en las aulas para conocer cómo se van recibiendo en cada caso las intervenciones - sea de los alumnos, sea de las maestras- que explican los procedimientos que emergen en sala de clase.

En este contexto también se elaboran algunas ideas que funcionan como criterios de evaluación de los conocimientos de los niños (por ejemplo, si los alumnos aplican los procedimientos "copiados" de sus compañeros en otros problemas en los que se cambian los números o las relaciones, podríamos confiar en que los han reconstruido) o puntos de apoyo para la intervención a partir de la estrategia de un alumno (por ejemplo, sería interesante desarrollar las ideas implicadas en las estrategias erróneas para considerar con los alumnos sus consecuencias y detectar contradicciones), o fundamentos para decidir -no siempre de la misma manera- respecto de qué grado de apertura alentar (por ejemplo, no siempre es conveniente llamar a que "hagan como puedan" para lograr que avancen, para que abandonen los palotes; a veces es necesario orientarlos un poco porque se abruman frente a un gran abanico de posibilidades; pero tienen que entender que no se buscan caminos únicos, el "hagan como puedan" ayuda a que resuelvan con control, entonces, ¿abrimos o marcamos un poco más lo que pueden hacer?). Es así como se va definiendo como dimensión de análisis el tipo de tratamiento que se da a los procedimientos de los alumnos y se introducen en ella diferentes variables que asumirán distintos valores según el contexto, la situación, los niños, los propósitos.

El análisis compartido sobre procedimientos de los niños y posibles intervenciones de una maestra con relación a un problema "de resta" similar al anterior pero propuesto en otro grado (Hay que juntar 280 pesos, ya tenemos 135, ¿cuánto falta juntar?) constituyó, desde nuestra perspectiva, un punto de inflexión en las consideraciones acerca del eje personal-grupal que se venían haciendo. En general, se recogieron diversidad de estrategias cuyo análisis ocupó un buen tramo de la reunión. Unos pocos niños sumaron las dos cantidades involucradas y en cierto momento la conversación se focaliza en ese procedimiento. La maestra comenta la dificultad para reconocer que los 135 pesos "son parte" de los 280 pesos que hay que juntar y que intervino con los niños recalcando esa relación (fijate que vos tenés que juntar 280, pero ya tenés algo, ¿cuánto tenés?). Es decir, ella subraya una complejidad relativa a este tipo de problemas en lugar de centrarse en la dificultad de los niños (que de hecho existe). Dado que, en el 
momento en que lo trae al espacio, los niños habían resuelto el problema en sus mesas pero no había ocurrido una instancia de puesta en común, se discute cómo organizarla:

M: No. Mi intención era que pasaran tres chicos que hicieron procedimientos variados, poder verlos, poder charlarlo con ellos, pero ahora con lo que estamos charlando.... Ahora, escuchando lo que decimos acá, tal vez pienso que podemos hablar antes de que pasen a hacer los procedimientos, quizás subrayar esto de que los 135 son parte de los 280 para todos, esto que sirvió para los que sumaron.

I: ¿Y eso lo querrías recuperar para todos?

M: Y, sí.

Entendemos que al haber subrayado en la discusión el valor de la intervención de la maestra que reconoce la complejidad de la relación implicada en el problema, se aprecia el interés de compartirlo con toda la clase y, ya no se piensa en términos del error de un alumno. Es decir, empieza a aflorar un criterio por el cual se interpreta que el error de un alumno puede estar mostrando la complejidad de una relación a construir y, en ese sentido, puede resultar interesante discutirlo con todos, hayan o no cometido errores. Este cambio de perspectiva ha sido, desde nuestro punto de vista, central en la constitución de este eje de análisis y consideramos que es una idea didáctica que puede generalizarse más allá de esta situación particular.

\section{3. ¿QUÉ SIGNIFICA AYUDAR A LOS ALUMNOS? ¿CÓMO RECONOCER SUS PROGRESOS?}

De una u otra manera estas preguntas fueron recorriendo las reuniones a la vez que su significado se fue transformando a medida que se profundizaban las discusiones. Los distintos tipos de ayudas que se pueden ofrecer a los alumnos, así como las reflexiones más generales que orientan su producción -y que nosotros insistíamos en explicitar como modo de construir fundamentos para la intervención- fueron temas traídos a la mesa de trabajo, siempre apoyados en los episodios de clase que las maestras aportaron. Los análisis también están atravesados en este caso por ese estado de transición al que con frecuencia, sobre todo en los primeros intercambios, refieren las maestras: se duda acerca 
del momento en que se debe ayudar a un niño, se interroga sobre su modo, su pertinencia.

De manera global podemos decir que, con el correr de las discusiones, se va contraponiendo la idea de guiar a los alumnos con la de promover el desarrollo de sus ideas para interactuar con ellas. Pero esta oposición tiene la intención de favorecer nuestro análisis y no resulta pertinente para interpretar el accionar de las maestras que aparece mucho más matizado y, sobre todo, muchas veces condicionado por el tipo de producción de los alumnos. Veamos.

Algunos docentes conciben la ayuda como una guía que se debe proporcionar a los alumnos. El modo en que se hace referencia a la acción de "guiar" parece sugerir que los alumnos con alguna dificultad estarían detenidos en algún punto del camino y la intervención docente aseguraría que continúen solos o acompañados- para arribar a una meta prevista. Todo ocurre como si se supusiera un recorrido anticipado por las maestras y la guía permitiría ubicar al niño en esa senda e indicarle las acciones a realizar para llegar.

Sin embargo, como intentamos señalar antes, este criterio no es homogéneo en el grupo de maestras y tampoco lo es para una misma maestra frente a situaciones diferentes. Es así como, por ejemplo, cuando se discute en el espacio alrededor de un problema de tipo aditivo, con números de dos cifras (45 y 17, referidas a panes y budines), planteado en un tercer grado, la maestra involucrada da cuenta de diferencias en la índole de ayuda que brindó a distintos grupos de alumnos:

- Frente a la producción de un niño que escribe el 4 (de 45) corrido hacia la izquierda respecto del 1 (de 17), la maestra, probablemente centrada en el algoritmo convencional, le plantea al alumno si acaso hay 400 panes. En este caso, le estaría atribuyendo al niño un intento vinculado al algoritmo convencional y, desde esa interpretación, lo estaría guiando para que corrija la posición del número 4 y lo ubique en la "columna" de las decenas. No obstante, ninguna traza del trabajo del alumno permite inferir que esté pensando en un procedimiento basado en el valor posicional.

- La maestra relata otro episodio referido al mismo problema: yo escuchaba que una de las nenas decía a sus compañeros de grupito "mirá, si yo me paro en el 45 y voy para allá" [refiriéndose a la banda numérica colgada en el pizarrón] entonces empezaron a contar para atrás. Después les pregunté "¿qué son los 45?, ¿por qué corriste para allá?". Y me contesta 
"estos (45) son los panes y éstos son los que se vendieron". Y por ahí escucho que en el grupito dicen "aah...".

- Un tercer episodio se diferencia, a nuestro juicio, de los anteriores: un grupito de alumnos sumó y yo les dije, "a ver, si yo tengo panes y los vendo, ¿voy a tener más o voy a tener menos?”. Y entonces una nena le dijo a la otra, "¿viste que teníamos que restar?".

Subrayamos que las tres intervenciones provienen de la misma maestra. En la primera, frente a la disposición "cuenta" que hace el alumno, ella parece dar por sentado que el niño se sitúa en el algoritmo convencional, no lo verifica y lo guía para que corrija. A raíz del segundo procedimiento, donde la alumna combina dibujo y conteo, tal vez con la intención de que se introduzcan elementos relativos al sentido de las operaciones - pero sobre la base de una resolución "correcta"- la maestra apunta a que la niña desarrolle sus ideas al pedirle que explicite sus razones. La docente registra -y relata en el espacio- que esta intervención irradió cierta comprensión en el grupito de niños, lo cual contribuye a poner sobre la mesa de trabajo que las explicaciones aportan comprensión aun cuando las resoluciones hayan sido correctas. La tercera intervención, orientada a que los alumnos revisen la operación que eligieron para resolver el problema, se basa en criterios más externos (si el resultado tiene que ser menor, no tiene sentido sumar) y no apunta a conocer por qué razones los niños optaron por sumar cuando debían restar.

El ejemplo nos permitiría inferir que no es pertinente atribuirle a un maestro una única posición con relación a sus modos de ayudar, sino más bien subrayar que las posibilidades de interpretación que ofrece la producción de los alumnos, así como el hecho de que sea "correcta" o "errónea", resultan componentes importantes para motorizar el tipo de ayuda.

Un avance en las discusiones llevará a hacer explícita la necesidad de asumir una posición en la que el docente reconoce la necesidad de apuntar al desarrollo de las ideas que subyacen a la producción de los alumnos -correcta o erróneacomo condición para interactuar con ellas.

A lo largo de las reuniones se van planteando estrategias de ayuda generales: pedirles a los niños que dibujen, que realicen un esquema de la situación, que subrayen los datos, que anoten. Asimismo, se reconoce que algunas intervenciones a veces son productivas y otras no lo son. Se alude a diversos recursos que no siempre constituyen soportes para orientar a los niños: grillas de números, descomposiciones, billetes y monedas, dibujos. 
El asunto que parece estar detrás de estas discusiones es el de articular algunas estrategias generales de ayuda con el contexto particular en el que deben insertarse -ahí el tipo de situación que los alumnos abordan y las producciones que realizan son determinantes-. Es decir, el tema que se empieza a instalar es que apelar a algunos procedimientos que tienen cierto grado de estandarización en las prácticas de enseñanza (que los niños subrayen, relean o dibujen) no le ahorra al docente el trabajo de hacer una interpretación respecto de las ideas que subyacen a la producción de los alumnos como para estar en condiciones de generar verdaderas interacciones.

M: Sí. Intentamos ver qué procesos hace y después ver cómo intervenimos nosotros para darle una validación, que tenga un significado.

Se generan condiciones para que podamos explicitar que las ayudas se basan en supuestos sobre las ideas de los niños, que es necesario tomar conciencia de ello y pensar a través de qué medios el docente puede confirmar sus interpretaciones sobre la producción de los niños y ayudarlos a validarla (Margolinas, 1993). Este es el punto que se empieza a hacer visible a raíz del análisis sostenido de episodios que las maestras aportan a las reuniones.

En este marco, el análisis de las producciones de los alumnos que los docentes aportaban a los encuentros permitió identificar que los niños, en numerosas oportunidades, ponían en juego relaciones que no siempre se reconocían fácilmente. La necesidad de que los alumnos desplieguen sus ideas y de entender qué hicieron o por qué no hicieron como condición para ayudarlos toma cuerpo. Este contexto resulta favorable para que se revisen ciertas estrategias instaladas en algunas docentes:

M: Yo no sé bien cómo se hace para que avancen. Yo, frente a un problema, iba dibujando lo que estaba leyendo. Yo, no ellos. Es que a veces tengo quizás esa tendencia a adelantarme yo al dibujo y quizás sería mejor que lo hagan ellos, sería otra instancia.

La estrategia de promover ayudas entre los alumnos también se analiza a partir de los aportes de las maestras. Es así como se conversa sobre la necesidad de enseñarles a los niños a ayudar a sus compañeros, sin decirles directamente el resultado. Entendemos que se conciben acá dos direcciones: para el alumno que tiene que elaborar una explicación y que se ve exigido de explicitar las 
relaciones matemáticas consideradas y, al mismo tiempo, para el alumno que recibe dicha explicación con la expectativa de las maestras de que cuente con más puntos de apoyo para tratar con el problema en cuestión.

Otra dimensión de análisis que se aborda se vincula a la producción de estrategias didácticas frente a respuestas de los alumnos que parecen no asumir como parte de la resolución la validez, pertinencia o coherencia de sus resultados. Al respecto, una maestra comparte su preocupación por el hecho de que varios de sus alumnos actúan, en algunas oportunidades, como si intentaran adivinar los resultados de los problemas, dando respuestas inconsistentes. En algunas reuniones se había discutido cómo contribuir a que los niños se responsabilicen por la validez de sus soluciones y, a raíz de esa cuestión, se había analizado la posibilidad de alentar a que los alumnos estimaran los resultados, dando un rango posible en el que podría encontrarse la solución.

Esta idea es recuperada por la maestra a la que estamos haciendo referencia, quien comenta en el espacio que les propone a sus alumnos un problema y les demanda anticipar cuál podría ser el resultado. Toma la decisión de confrontar a los "chicos con los propios chicos", es decir, los resultados encontrados con sus propias anticipaciones. Está promoviendo de esta manera que los alumnos se hagan una primera representación que -se analiza en el grupo colaborativoactuaría como marco y punto de apoyo para la resolución de los niños. La confrontación entre lo anticipado y lo obtenido a la vez se transforma en un soporte a partir del cual la maestra puede organizar una discusión. Entendemos que esta estrategia se sostiene en un supuesto: la contradicción en la que incurrirían los niños al constatar las diferencias con lo anticipado es una fuente de producción en la medida en que se generan condiciones para intentar explicarla. Una hipótesis a seguir explorando surge a partir de este episodio: apelar a la anticipación de los alumnos es un tipo de ayuda que en sí misma moviliza ideas de los niños que pueden considerarse como base para la resolución y la validación de los resultados. Se generan condiciones para la producción de herramientas que hagan posible la validación por parte de los alumnos; estas condiciones se ligan a tomar el problema que se resuelve como objeto de análisis.

En el apartado precedente, referimos un episodio en el que una maestra, a raíz de un problema de resta, interviene a nivel de las relaciones involucradas. Lo recuperamos ahora para analizarlo desde el punto de vista de las ayudas. "Para el problema en cuestión (Hay que juntar 280 pesos, ya tenemos 135, ¿cuánto falta juntar?), la docente interactúa con un niño que sumó las dos cantidades y subraya la relación parte-todo implicada en el problema (fijate que 
vos tenés que llegar a tener 280, pero vos ya tenés algo, ¿cuánto tenés?). Entendemos que la maestra asumió que este alumno no estaba identificando la relación existente entre los datos y, desde esa interpretación, interactúa con el alumno. Intervenir a nivel de las relaciones implicadas más que de las acciones a realizar supone, desde nuestro punto de vista, un cambio en los sentidos de las ayudas a los niños que este grupo colaborativo logró producir.

Se analizan en el grupo episodios en los que se hace visible que, al tiempo que se ayuda a los niños, se reconocen sus progresos. Un lema que se hace explícito envuelve estas discusiones: para entender las ideas de los niños hay que intervenir y a la vez esa comprensión da lugar a nuevas intervenciones. En este contexto la ayuda podría entonces interpretarse como una mediación entre ciertos conocimientos de los niños y otros, que constituyen parte de la intención docente, a ser elaborados, modificados, transformados, generalizados.

En una reunión de balance sobre los avances del grupo colaborativo, la cuestión de las ayudas a los niños tuvo un lugar relevante:

M1: Más allá de que esté la hoja en blanco, que la intervención docente lo ayude a aquel que se equivocó...

M2: Igual, ¿qué es la ayuda? Porque es eso lo que uno se está planteando, ¿qué es ayudarlo?

[...]

M2: Sí, lo estoy pensando, revisar qué es ayudar porque quizás nosotros también los ayudábamos. Pero, ¿cómo los ayudábamos a que se metan?

M3: Sí, yo creo que desde todo punto de vista, ayudarlo para que pueda avanzar, pero también ayudarlo a que quiera trabajar, porque si nos quedamos con el que es rápido para las matemáticas y al que le cuesta más o se muestra paralizado... M: [refiriéndose a un alumno] él fue el que explicó cómo se hacía, entonces es como que la ayuda no es sólo para el alumno, sino también entender lo que el chico está pensando y puede hacer, lo ayuda a él y nos ayuda a nosotros...

M3: Y ayuda a toda la clase.

$[. .$.

M4: Yo creo que es fundamental no perder de vista a los chicos, y los procesos de los chicos.

Sostener la discusión sobre "las ayudas" a lo largo del tiempo, problematizarlas, hizo posible que el espacio de trabajo quedara más cerca de concebirlas como favorecedoras del desarrollo de las ideas de los alumnos, como base para la 
interacción con ellos, que de entenderlas como guías para la acción. En esta línea se conceptualiza la relación entre algunas estrategias didácticas generales y la necesaria producción por parte del docente que implica su contextualización en un caso específico de intercambio con los alumnos. Asimismo, se identifica que son necesarias en diferentes momentos: para entrar en el juego, para explicitar o reconocer relaciones, para avanzar en ciertos recursos, para explicar decisiones, para provocar confrontaciones y producir ideas a partir de ellas.

Si bien, nos parece claro identificar a las ayudas como intervenciones didácticas específicas, nos hemos preguntado qué las distingue de otras intervenciones. Esta demarcación resulta difusa, cualquier intervención docente podría ser pensada en sentido amplio como una ayuda. Sin embargo, cuando las maestras las han traído al espacio, fue para considerar situaciones que de alguna manera les resultaban extremas. A su vez, fueron estas situaciones las que hicieron más fácilmente visibles condiciones del funcionamiento de la clase en general, amplificando fenómenos que suelen correr más silenciosamente en el aula.

\section{LAS MEDIACIONES NECESARIAS PARA LA UTILIZACIÓN DE RECURSOS POR PARTE DE LOS ALUMNOS}

Las docentes proponen en sus aulas diferentes tipos de problemas para que los niños movilicen sus propios conocimientos, con la expectativa de que sean punto de apoyo para arribar a las relaciones matemáticas que ellas se plantean enseñar. Sin embargo, muestran preocupación porque consideran que unos cuantos niños despliegan procedimientos muy básicos: dibujos, palitos... Cómo promover que los alumnos dejen de usar dibujos y reconozcan y usen operaciones aritméticas, es considerado un problema de enseñanza sobre el que proponen discutir. Se reconoce que este pasaje de dibujos a números y, más en general, del uso de recursos considerados muy elementales a otros más elaborados, no es automático.

Se comparten en el espacio colaborativo diferentes intervenciones que proponen las maestras en sus aulas y que evidencian la intención de favorecer progresos en los recursos que utilizan sus alumnos: apelar al uso de billetes como soporte del conteo, alentar la descomposición aditiva de números como puente hacia la realización de cálculos, promover el uso de tablas de números ordenados para impulsar la coordinación entre numeración hablada (conteo) y representaciones escritas de los números e incluso sugerir explícitamente el uso 
de escrituras numéricas en lugar de palitos o dibujos. Se analiza que, al poner en funcionamiento algunos de estos recursos, se hace visible para las docentes la necesidad de otros en los que no habían pensado (les propuse contar billetes y mientras lo hacían me di cuenta de que muchos no sabían contar de 5 en 5 o de 10 en 10, lo cual me obligó a hacer una actividad para eso). Episodios como éstos muestran simultáneamente la atención que las maestras prestan al modo en el que los niños responden a las actividades y la disposición que tienen a proponer, sobre la marcha de la clase, otras actividades mediadoras cuando reconocen la ausencia de ciertos recursos por parte de los niños. De manera que la relación entre problemas y recursos está presente en el accionar y en la conciencia de las docentes y la discusión en el espacio colaborativo va a permitir profundizarla. Veamos.

En distintas oportunidades varias maestras hacen referencia a que proponen la realización de descomposiciones aditivas de los números con la expectativa de facilitar la entrada en el cálculo:

M: Ahora quiero ver si trabajando mucha composición y descomposición de números, y ahí me planteaba si podían hacer cálculos.

Parecía haber un supuesto en esas expectativas: si, como está muy documentado, la descomposición aditiva basada en la organización decimal de las escrituras numéricas es un recurso que los niños usan para sumar y restar, un paso previo para promover el pasaje al cálculo sería que se ejerciten en la descomposición aditiva de los números en "cienes, dieces y unidades". Sin embargo, las mismas maestras constatan que apelar a las descomposiciones, los billetes y otros recursos no produce de manera inmediata que los alumnos se "vuelquen" al cálculo, lo cual les provoca cierta incomprensión y desconcierto. La discusión en el espacio sobre este asunto contribuye a problematizar los supuestos que subyacen al punto de vista según el cual hacer descomposiciones habilita, de manera general, su uso en el cálculo.

I: Lo que está apareciendo es que las sumas con dieces, cienes, miles no es algo que los chicos usan solos.

Varias M: No.

M: Yo lo que veo inclusive es que la descomposición la hacemos para enseñar la numeración, porque se hace, lo hacemos, pero después para volcarlo en el repertorio 
de operaciones no lo hacemos (...) y no aprovechamos, no utilizamos este sumar cienes, dieces y después terminamos haciendo el procedimiento tradicional.

I: Si no se enseñan cómo juegan en los cálculos...

[...]

M: Si después de haber aprendido a descomponer los números, los van a poder llevar a resolver un problema.... con estas dos cuestiones a mí me abren más incógnitas... ¿cómo hago para que aprendan a descomponer?, ¿cómo llevan eso a la resolución del problema?

A partir de estas intervenciones en las que se pone de manifiesto que no es lo mismo comprender el funcionamiento de un recurso que usarlo en la resolución de una cuestión específica, se empieza a desarrollar una discusión en la que se plantean dos grandes puntos: a) los recursos, para que sean tales, no deberían aislarse de los problemas para los cuales pueden constituirse en procedimientos de resolución y, b) tal vez sea necesaria la intervención explícita de las docentes para movilizar el uso de estrategias, intervención que muchas maestras parecen eludir por temor a invadir el ámbito de construcción autónoma del niño ${ }^{9}$. En esta discusión se puede apreciar cómo se van entramando de manera coordinada los aportes de investigadores y docentes: al intentar explicar un hecho desconcertante para las maestras con el que se encuentran en sus prácticas (los niños no ponen en juego un recurso ejercitado), se vuelve significativa una idea más general según la cual la movilización de un recurso es inescindible de su aprendizaje "en situación".

Como síntesis de estas discusiones se identifica que el avance en los recursos que producen los alumnos requiere, más que de una secuencia lineal de adquisiciones, de un entramado de relaciones matemáticas en el que los niños elaboran de manera coordinada ideas cercanas entre sí: relaciones aritméticas implicadas en las escrituras numéricas, uso de esas relaciones para el cálculo, vínculos entre contar y operar.

En la misma línea -así lo entendemos- de lo ocurrido entre descomposición de los números y uso de esta estrategia para calcular, es decir en la línea de problematizar las diferencias entre las expectativas de las maestras con relación

9 En las distintas experiencias de trabajo colaborativo que hemos llevado a cabo en nuestra investigación nos hemos encontrado frecuentemente con "temores" de este tipo por parte de las docentes. Entendemos que provienen de una interpretación del constructivismo - probablemente devenida en un cierto "sentido común" - en la que se sostendría que si el docente "dice", el alumno "no construye". Los análisis del grupo colaborativo han permitido visibilizar y poner en cuestión esta idea. 
al accionar de los chicos y lo realmente sucedido, ubicamos los análisis realizados en el grupo con relación a un recurso didáctico, frecuente en las prácticas escolares, como es el de "bajar los números" cuando los niños encuentran dificultades para resolver un problema.

En efecto, nuevamente en el marco del trabajo con relación a la resta, una docente comenta que propuso a sus alumnos un problema cuyas cantidades eran números de tres dígitos y que su abordaje pareció paralizar a unos cuantos niños. La docente comparte en el espacio que conversó estas dificultades con una compañera (también integrante del grupo) que le sugirió proponer un problema similar con números de dos dígitos. Esta decisión parece sostenida en el siguiente supuesto: si los alumnos tratan el problema con números más chicos, los recursos y relaciones que allí desplieguen servirían como punto de apoyo para tratarlo con números más grandes.

Varios docentes comparten este modo de mediar: bajar los números para que los alumnos puedan atrapar las relaciones matemáticas que subyacen al problema. Sin embargo, las mismas maestras reconocen que no siempre esa mediación funciona y que al retomar el problema con números más grandes los alumnos no reutilizan las relaciones que sí movilizaron con un rango de números menor. La docente que inicialmente trajo la cuestión al grupo analiza que tuvo necesidad de realizar una puesta en común, desmenuzando las relaciones entre el primer problema y el segundo (con números menores) para que los alumnos pudieran abordarlo. Notemos que los vínculos entre contar para atrás, recurso que utilizan los niños cuando los números son relativamente bajos, y restar tampoco eran evidentes para muchos alumnos y que el análisis de los dos problemas en conjunto permite tematizar.

Estos dos episodios que acabamos de compartir nutrieron al espacio de un asunto que continuó siendo tema de discusión: para ser reutilizadas por los alumnos, las mediaciones a las que recurren los docentes requieren la elaboración de relaciones matemáticas -nuevas para los chicos- que muchas veces el docente debe aportar. De manera transversal, la pertinencia de la intervención docente se vuelve a poner sobre la mesa como tema de debate.

\section{CONCLUSIONES}

Estado de transición e incertidumbre fueron palabras claves para caracterizar inicialmente el posicionamiento del grupo de docentes de la Escuela 30 al 
comenzar el trabajo colaborativo. Poco a poco, las discusiones fueron profundizando -desarrollando- el contenido de estas claves al tiempo que se iba haciendo visible la complejidad de la enseñanza cuando existe la intención genuina de dar lugar a las ideas de los alumnos en las discusiones matemáticas del aula. El accionar docente se problematiza en términos de dimensiones de análisis a tener en cuenta como soporte de las decisiones de enseñanza y también de nuevas disposiciones acerca de las cuales los integrantes del grupo no teníamos conciencia plena. Asimismo, el examen de los episodios de las aulas contribuye a poner de manifiesto la gran cantidad de pequeñas relaciones matemáticas constitutivas de los conocimientos que los alumnos deben elaborar y que sólo se pueden reconocer cuando se estudian en profundidad las interacciones en sala de clase en términos de relaciones matemáticas implicadas y, de interpretaciones que las comandan y se hipotetiza acerca de cómo retomarlas e inscribirlas en conceptos más generales.

Los ejes de análisis que fueron emergiendo (intervenciones grupales o personales, tipos de ayudas, mediaciones necesarias para el progreso de los alumnos) en este grupo se fueron reconociendo a partir de considerar como asunto de discusión en el espacio colaborativo las dudas de los distintos participantes, los desconciertos generados por las distancias entre expectativas (de los docentes) y hechos (de las aulas), las diferentes interpretaciones sobre aquello que en principio se manifestaba como incomprensiones de los alumnos, la confrontación entre las distintas decisiones tomadas por las maestras frente a situaciones similares. De ninguna manera pretenden abarcar todas las dimensiones posibles de análisis de la enseñanza de la matemática y, muy probablemente, en otro grupo emergerían otras líneas de análisis en función de los problemas que reconocen los docentes.

Para cada uno de los ejes tratados en este artículo, formulamos a modo de síntesis una pregunta que estructura su contenido: ¿en qué sentido las elaboraciones de un alumno (o de un grupo) -sean correctas o erróneas, hayan apelado o no a modos convencionales de escritura- contribuyen a ampliar las consideraciones sobre el objeto de enseñanza-aprendizaje para el conjunto de la clase (alumnos y docente)?; ¿cuáles son los distintos niveles de intervención que contempla la intención de ayudar a los alumnos?; ¿qué relaciones -qué condiciones- se pueden entablar entre la consideración de un procedimiento, una relación, una forma de representación entendidos como medios de resolución y su utilización efectiva? Notemos que son preguntas que, lejos de derivar en un conjunto de reglas de acción, abren perspectivas de análisis. Las 
respuestas que se van encontrando, ligadas al contexto particular en el que emergieron, contribuyen a la elaboración de criterios para la acción. Estos criterios, ligan una decisión (de enseñanza) con sus razones (las que se han elaborado en el caso particular) y, justamente por no constituir reglas, no predeterminan el accionar docente ni estandarizan las intervenciones posibles de los maestros (Bednarz, 2013).

Las elaboraciones realizadas a raíz de cada uno de los ejes fueron delineando distintos tipos de intervenciones docentes y a la vez este proceso de problematización dio lugar a tomar conciencia de que la posibilidad de integrar a la enseñanza las ideas de los alumnos está ligada a una disposición docente ( a ir a buscar las ideas de los niños, a interpelarlas, a promover su desarrollo) que, lejos de depender exclusivamente de la voluntad, se va construyendo y consolidando en la medida en que las interacciones en sala de clase se constituyen en asunto de estudio. De manera solidaria con estos posicionamientos se fue registrando cada vez con mayor precisión que los objetos de enseñanza se modifican al proponer esta integración. Como síntesis de estas cuestiones, podríamos decir que la disposición a incorporar las ideas de los niños y la conciencia de que se modifica el objeto de enseñanza se constituyen en dos pilares de la conceptualización que tiene lugar en el espacio.

A medida que el grupo colaborativo consolidaba su constitución, las maestras fueron adoptando una posición exploratoria desde la que proponían estrategias didácticas para abordar problemas que reconocían en la enseñanza, las desarrollaban en sus aulas y recogían material para considerar el funcionamiento de su implementación en el espacio colaborativo. El análisis en el grupo permitió revisar y ajustar las estrategias elaboradas, identificar relaciones y variables que intervienen, tomar conciencia de que el examen minucioso de los sucesos de la clase contribuye a reformular las situaciones que se proponen para los alumnos con la intención de lograr con mayor solidez los objetivos. La idea de que las estrategias que se planean y las intervenciones que se realizan obedecen a supuestos que el análisis permite explicitar va haciendo visible una postura según la cual los proyectos de enseñanza se interpretan como hipótesis a explorar en las aulas y esto fortalece -así lo entendemos- la posición de las maestras como productoras de conocimiento. Fue así como el espacio de colaboración fue tornándose en una referencia explícita para las decisiones didácticas que las maestras tomaban en las clases al tiempo que las interacciones (maestro-alumno-saber) se constituían en la referencia que nutría la construcción del espacio. Esta dinámica hizo posible objetivar cada vez más las 
discusiones y fue fundamental en el proceso de reconfiguración conceptual de la enseñanza.

Detengámonos en el grado de generalidad que tiene esta reconfiguración y, en qué sentido, podríamos considerar que los conocimientos producidos en el espacio colaborativo podrían retomarse en procesos de formación docente más descontextualizados. Desde nuestro punto de vista, la producción central se refiere a que los ejes de análisis contribuyen a tomar conciencia de dimensiones a considerar y no prescriben acerca de cómo deben plasmarse en cada caso. Digamos también, que estos ejes, surgidos del examen analítico de episodios de las prácticas de enseñanza que las mismas docentes recortaron, no se contraponen en absoluto a otras dimensiones de análisis provenientes de marcos teóricos que venimos sosteniendo los investigadores y que están implicados en los aportes que hacemos al espacio de trabajo; por ejemplo, la consideración de las relaciones entre los problemas que se plantean y las elaboraciones a las que dan lugar, o los modos que adquiere la validación de las resoluciones en la clase o las maneras en las que se distribuyen las responsabilidades por la validez de las afirmaciones, todos estos ejes delineados en el marco de la Teoría de Situaciones Didácticas de Brousseau (2007)). Queremos enfatizar que se trata de dimensiones analíticas que provienen de diferentes modos de problematizar el conocimiento y que no son -pensamos- incompatibles entre sí: uno toma como referencia principal las prácticas de producción de conocimiento matemático y otro, las prácticas de enseñanza de la matemática en el contexto escolar. Una hipótesis que se asoma a partir de nuestro estudio y que necesitará ser explorada con profundidad es que la coordinación entre estas diferentes fuentes de problematización encuentra un lugar posible de realización en el trabajo colaborativo entre investigadores y docentes. Se trata de un trabajo teórico no desarrollado aún.

El sostener las discusiones durante un período prolongado ha hecho posible retomar aspectos tratados en un cierto momento y resignificarlos a raíz de nuevos asuntos de enseñanza, tanto para encontrar elementos comunes como para establecer diferencias. La misma idea de evolución de los aprendizajes, que fue un hilo estructurador de las reuniones, comporta una dimensión temporal que excede ampliamente el análisis de episodios puntuales. Asimismo, la necesidad de respetar los estilos de cada docente, al tiempo que se asegura a través de la discusión la construcción de acuerdos que comprometen a todo el equipo estuvo presente, sobre todo en las reuniones de balance. Vemos entonces que las dimensiones temporal e institucional se instalan en el grupo colaborativo, 
haciendo visible la noción del aula como sistema abierto y de la enseñanza como hecho público, entablando un diálogo entre cada episodio y proyecto $y$, otorgando sostén a la necesidad de la construcción de lo común.

Como hemos señalado, aunque no fue el foco de este artículo, nuestro grupo estudia la constitución de la colaboración. La perspectiva de construcción de simetría entre investigadores y docentes propuesta por Sensevy (2011), a la que ya hemos hecho referencia, ha tenido un alto valor heurístico en nuestro proceso de producción. Reconociendo su importancia, queremos señalar que consideramos necesario matizar esta noción para no caer en una visión que nos haga conceptualizar a cada uno de estos dos grupos como homogéneos (los docentes y los investigadores), lo cual podría comportar el riesgo de que pasaran inadvertidas las diferentes perspectivas de los integrantes de cada grupo, que tan ricas han resultado para potenciar los procesos de discusión.

Finalmente, queremos volver una vez más a la idea de incertidumbre con la que abrimos este artículo. Las maestras de este grupo, al compartir su experiencia en la que han entramado sus análisis, su compromiso y sus preocupaciones, nos han enseñado que existe un punto irreductible (que una de las maestras nombró de manera sabia como caja negra): nunca terminaremos de entender las razones por las que algunos niños no estabilizan las relaciones que parecían comprendidas, no reutilizan las ideas tal como esperábamos o no terminan de entrar en el juego de conocimiento que les proponemos. Esa zona de misterio requiere seguir siendo explorada y sus enigmas nos reúnen y nos motorizan.

\section{REFERENCIAS}

Bednarz, N. (2004). Collaborative research and professional development of teachers in mathematics. ICME 10. (U. o. Monreal, Ed.) Montreal, Canada.

Bednarz, N. (2013). Regarder ensemble autrement: ancrage et développement des rechreches collaboratives en éducation au Québec. In N. Bednarz, Recherche collaborative et pratique enseignante. Regarder ensemble autrement (pp. 13-29). Paris: L'Harmattan.

Bednarz, N., \& Proulx, J. (2010). Développement professionelle des enseignants en mathématiques. In B. De Lièvre, A. Braun, V. Carelle \& W. Lahaye, Éducation et formation. Travail en communautés, collaboration et partenairiats pour le développement professionelle des enseignaints, e-293 (pp. 21-36). Bélgica: Université de Mons. 
Brousseau, G. (1998). Théorie des situations didactiques. Grenoble: La Pensée Sauvage.

Brousseau, G. (2007). Iniciación al estudio de la teoría de las situaciones didácticas. Buenos Aires: Libros del Zorzal.

Del Rey, A. (2012). Las competencias en la escuela. Una visión crítica sobre el rendimiento escolar. Buenos Aires: Paidós.

Delprato, M. (2013). Condiciones para la enseñanza de la matemática a adultos de baja escolaridad. (Tesis de doctorado no publicada), Universidad Nacional de Córdoba, Argentina. Retrieved from https://ansenuza.unc.edu.ar/comunidades/handle/11086.1/809

Fisher, M. (2016). Realismo capitalista. Buenos Aires: Caja Negra.

Lave, J. (2001). La práctica del aprendizaje. In S. Chaiklin \& J. Lave, Estudiar las prácticas. Perspectivas sobre actividad y contexto (pp. 15-45). Buenos Aires: Amorrortu editores.

Margolinas, C. (1993). De l'importance du vrai et du faux. Grenoble: La Pensée Sauvage.

Pastré, P., Mayen, P., \& Vergnaud, G. (2006). La didactique professionnelle. Revue française de pédagogie (154), 145-198.

Proulx, J. (2013). Réflexions épistémologiques sur la recherche collaborative en didactique: possibilités et excès. In N. Bednarz, Recherche collaborative et pratique enseignante. Regarder ensemble autrement (pp. 327-349). Paris: L'Harmattan.

Robert, A. (2003). De l'idéal didactique aux déroulements réels en classe de mathématiques : le didactiquement correct, un enjeu de la formation des (futurs) enseignants (en collège et lycée). (I.N. Pédagogique, Ed.) Didaskalia (22).

Sadovsky, P., Quaranta, M.E., Itzcovich, H., Becerril, M.M., \& García, P. (2015). La noción de relaciones entre cálculos y la producción de explicaciones en la clase de matemática como objetos de enseñanza. Su configuración en el marco de un trabajo colaborativo entre investigadores y docenetes. Educación Matemática, 27(2), 7-36.

Sadovsky, P., Itzcovich, H., Quaranta, M., Becerril, M., \& García, P. (2016). Tensiones y desafíos en la construcción de un trabajo colaborativo entre docentes e investigadores en didáctica de la matemática. Educación Matemática, 28(3), 9-29.

Sadovsky, Quaranta, Itzcovich, Becerril \& García. (2015). Producción matemático-didáctica: una experiencia de planificación colaborativa entre maestros e investigadores. En A. Pereyra, \& D. Fridman, Prácticas Pedagógicas y Políticas Educativas. Investigaciones en el territorio bonaerense (pp. 221-250). Gonnet: Unipe: Editorial Universitaria.

Sadovsky, Quaranta, M., García, P., Becerril, M., \& Itzcovich, H. (2018). Los análisis de las intervenciones docentes en el marco del trabajo colaborativo entre investigadores y docentes como puente hacia la inclusión educativa. En A. Pereyra, S. Bernatené, \& 
D. Fridman, Los desafíos de la educación inclusiva. Actas del 40 Coloquio Internacional sobre Educación Inclusiva. (págs. 614-621). Buenos Aires: Unipe: Editorial Universitaria.

Sensevy, G. (2011). Le sens du savoir. Bruselas: De Broeck.

\section{PATRICIA SADOVSKY}

Domicilio: Solier 4869. Villa Domínico

Avellaneda, Buenos Aires

CP 1874

Teléfono: +5491135897692 